\title{
Attitudes and Perceptions among Dublin International Foundation College Students towards Taking Conventional Medications and Herbal Medicines
}

Abdullah T. Eissa.

\begin{abstract}
Background: The use of both conventional medications and herbal medicines has increased recently. Consequently, the chance of misusing medications has also increased, leading to unwanted complications and economic burdens. This study examined the attitudes and perceptions of international students at Dublin International Foundation College (DIFC) towards conventional medication and herbal medicine. Methods: A cross-sectional quantitative method was used to collect information from international students at DIFC. A total of 85 questionnaires were distributed to the students, and 54 completed questionnaires were received from them. Chi-square test was used to examine the possible relationships between students' background, gender and region of origin and their attitudes and perceptions toward using conventional medications and herbal medicines. Results: Participants from different regions of origin showed significant differences in the responses to questions about informing their healthcare providers about their drug allergies. While most students did not usually mix herbal medicines with either conventional medications or other types of herbs, students aged between 21 and 25 were more likely to combine different types of medications compared to students from other age groups. No significant differences were observed in relation to students' educational backgrounds and gender. Conclusion: This study demonstrated the need of educating students from different regions and backgrounds about the use of conventional medications and herbal medicines. Information regarding the proper use of medications and the dangers of drug interactions should be included in the curricula of formal education and disseminated to the public using culturally sensitive strategies.
\end{abstract}

Keywords: Herbal Medicine; Complementary Therapies; Pharmaceutical Preparations; Health (Source: MeSH-NLM).

About the Author: Abdullah Talat Eissa is a final-year medical student at king Saud bin Abdulaziz University for Health Sciences, Riyadh, Saudi Arabia. He is also a pharmacist who graduated from king saud University in 2010

\section{Submission: Apr 5, 2015 \\ Acceptance: Aug 31, 201 \\ Publication: Dec 37, 2015}

and herbal medicines have yet to be extensively studied. This

\section{Introduction}

The use of conventional medications has been rising over the last few decades. In the United States, nearly half of Americans used at least one prescription medication in the past month.' Increased prescription drug use is associated with increased misuse of medications, which may lead to more complicated situations or even mortality. The misuse of conventional medications can have a negative impact on the economic system, leading to increased health care costs.

At the same time, some patients prefer alternative or herbal medicine. Alternative medicine or complementary medicine are used interchangeably to refer to health practices that are not part of a country's own tradition and are not integrated into the dominant health care system. Herbal medicine is an alternative medicine approach which uses parts of plants, other plant materials, or their combinations as active ingredients for treatment. The use of herbal medicine has increased significantly over the last 10 years. ${ }^{2}$ This may be due to consumers' belief that herbal medications have fewer side effects, are cheaper and more effective than conventional medications. ${ }^{3,4}$

Despite the increased popularity of conventional medications and herbal medicines, many people lack the appropriate knowledge, attitudes and behavior for proper use of medicines, leading to untoward negative outcomes. The attitudes or perceptions of international students toward using conventional medications study thus aimed to find out the attitudes and perceptions of international students in Dublin International Foundation College towards taking conventional medications and herbal medicines. As the college attracts international students from different regions and backgrounds, this study could help to identify how students' different backgrounds could affect their attitudes and perception towards conventional and alternative medicine.

\section{Methods}

This cross-sectional study used a self-administered questionnaire, which was distributed to all Dublin International Foundation College (DIFC) students $(n=85)$. The study lasted two months, from mid-June to mid-August 2013. As the participants were non-native English speakers, the questions were formulated in simple English, and the DIFC staff who distributed the questionnaire provided explanation of words which participants found to be difficult. The survey was anonymous and confidential, and permission was obtained from the participants prior to questionnaire administration. Data collected was stored securely in the researcher's computer using code numbers to ensure confidentiality during data analysis. The study protocol was approved by the institutional review board (IRB) of DIFC.

The participants of this study were students who came from different countries, which are Saudi Arabia, China, Libya, Malaysia, Oman and Nigeria. As the expected count was less than 
five in certain cells if country was used as the categorical variable and thus making Chi-square test inaccurate, the participants were classified based on their geographical regions. Saudi, Libyan and Omani students were identified as Middle East students because they have similarity in many habitual characteristics, such as language and Arabic traditional customs. Chinese and Malaysians were analyzed as Asians, as they came from countries located in the Far East region of the Asian continent. Nigeria represented Africa in the data analysis.

The survey was developed by the author after discussing the most common controversial medical issues among their international students with the college's academic staff. Afterwards, the questionnaire was structured and adjusted to be easily understood by non-native English-speaking students. The questionnaire consisted of two pages. The first page included instructions for filling out the questionnaire and items for collection of demographic data (gender, age, nationality and highest educational background). The second page consisted of 12 questions. There were six questions regarding the attitudes and perceptions of the students toward conventional medications, four questions towards herbal medicine, and two questions about their attitudes and perceptions toward gaining information about medications. Most of the questions were based on a five-point Likert scale ("never", "rarely", "sometimes", "usually" and "always"), except for three questions which allowed for three responses ("yes", "no" and "I am not sure").

After collecting the answered questionnaires manually from the participants, the data was analyzed statistically using SPSS 22.0 software for Windows. Chi-square test, with the level of significance set at $p<0.05$, was used to compare categorical data and examine the relationship between students' background, gender and nationality and their attitudes and perceptions toward using conventional medication and herbs.

\section{Results}

Out of the 85 questionnaires which were distributed to the students, 54 completed questionnaires were received from them (response rate $=63.5 \%$ ). In this study, the age of respondents ranged from 16 to 30 years, and the mean age was $22.13+3.79$ years old. There were 28 females and 26 males. The distribution of participants by region of origin was as follows: 22 students were from the Middle East, 23 students were Asians, and 9 students came from Africa. The students had different educational backgrounds; there were 11 students with bachelor degrees, 20 students with college diplomas and 23 students with high school education.

The responses to all 12 questions of the survey were not significantly associated with gender and educational background. The attitudes and behaviors of the participants measured by the first 10 questions of the survey were not significantly different in relation to their age. Nevertheless, responses to question number 11 (which was about mixing herbal medicine with prescribed medication) and question number 12 (which was about mixing more than one type of herbal medicine) were significantly different among students from different age groups ( $p<0.001$ and $p$ $=0.001$, respectively). Most students answered "never" and "sometimes" for these two questions, and students aged between 21 and 25 were more likely to combine different types of medications compared to students from other age groups (Table 1).

Most students answered that they always tell their doctors and pharmacists about their drug allergies (Table 2). Around $52 \%$ of the Middle Eastern students and $48 \%$ of the Asian students answered that they always reported their drug allergies to doctors and pharmacists, while a higher percentage ( $75 \%$ ) of Africans told their healthcare providers about their allergies ( $p=$ 0.022). Majority of the Africans and Asians answered that they "rarely" and "sometimes" took medications from their friends, while most of the Middle Eastern students did that "rarely" ( $p$ $=0.081$ ). Most of the respondents were not sure and disagreed with the statement that herbal medicine can treat all diseases, regardless of their regions of origin $(p=0.05)$. In addition, the vast majority of the Africans said that they never mix prescribed medications with herbs, while most Middle East and Asian students answered that they "never" and "sometimes" did that.

\section{Discussion}

The proper use of conventional medication is well-defined because there are many important guidelines and regulations to direct patients on how to administer their medications, including guidelines on the reasonable use of medication. ${ }^{5}$ On the other hand, the consumers' knowledge and their needs for alternative medicine differ from region to region and from country to country. ${ }^{6}$ In Nigeria, $85 \%$ of people knew how to use herbal medicine, ${ }^{7}$ and all of the undergraduate pharmacy students (100\%) at University of Sierra Leone were aware of some

Table 1. Students' Responses to Questions 11 and 12 According to Their Age.

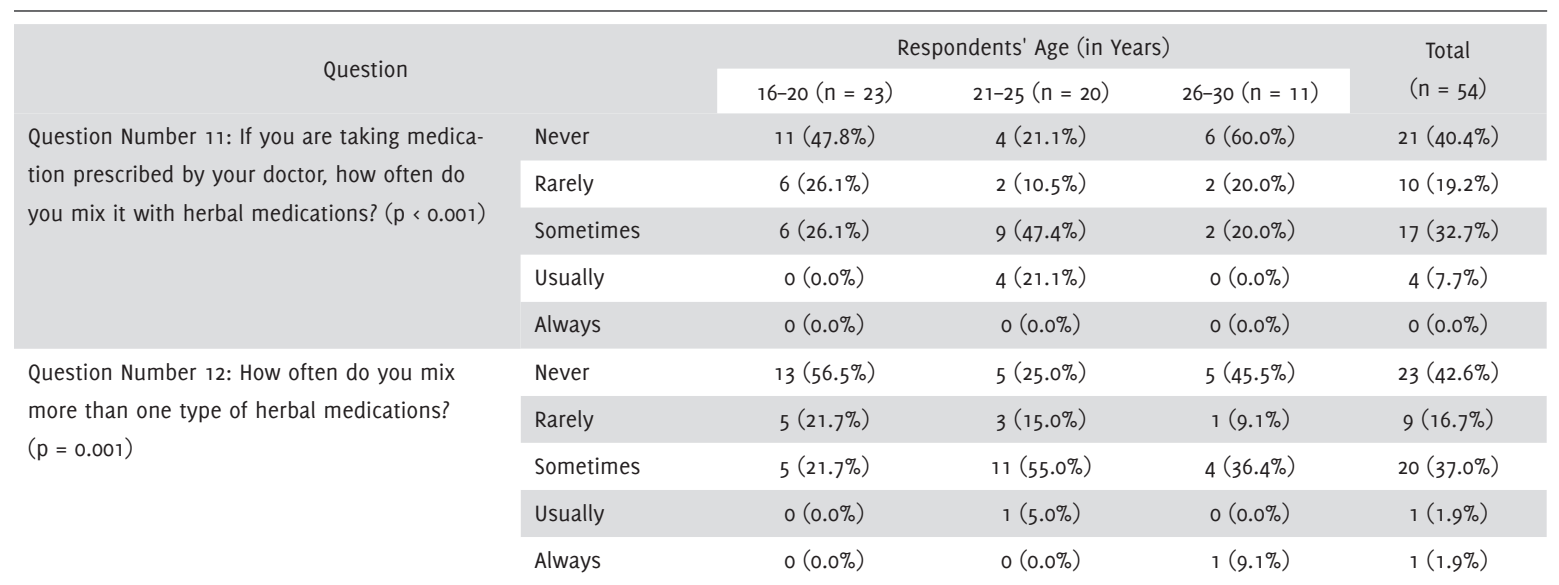


Table 2. Students' Responses to Questions 3, 7, 9, and 11 According to Their Regions of Origin.

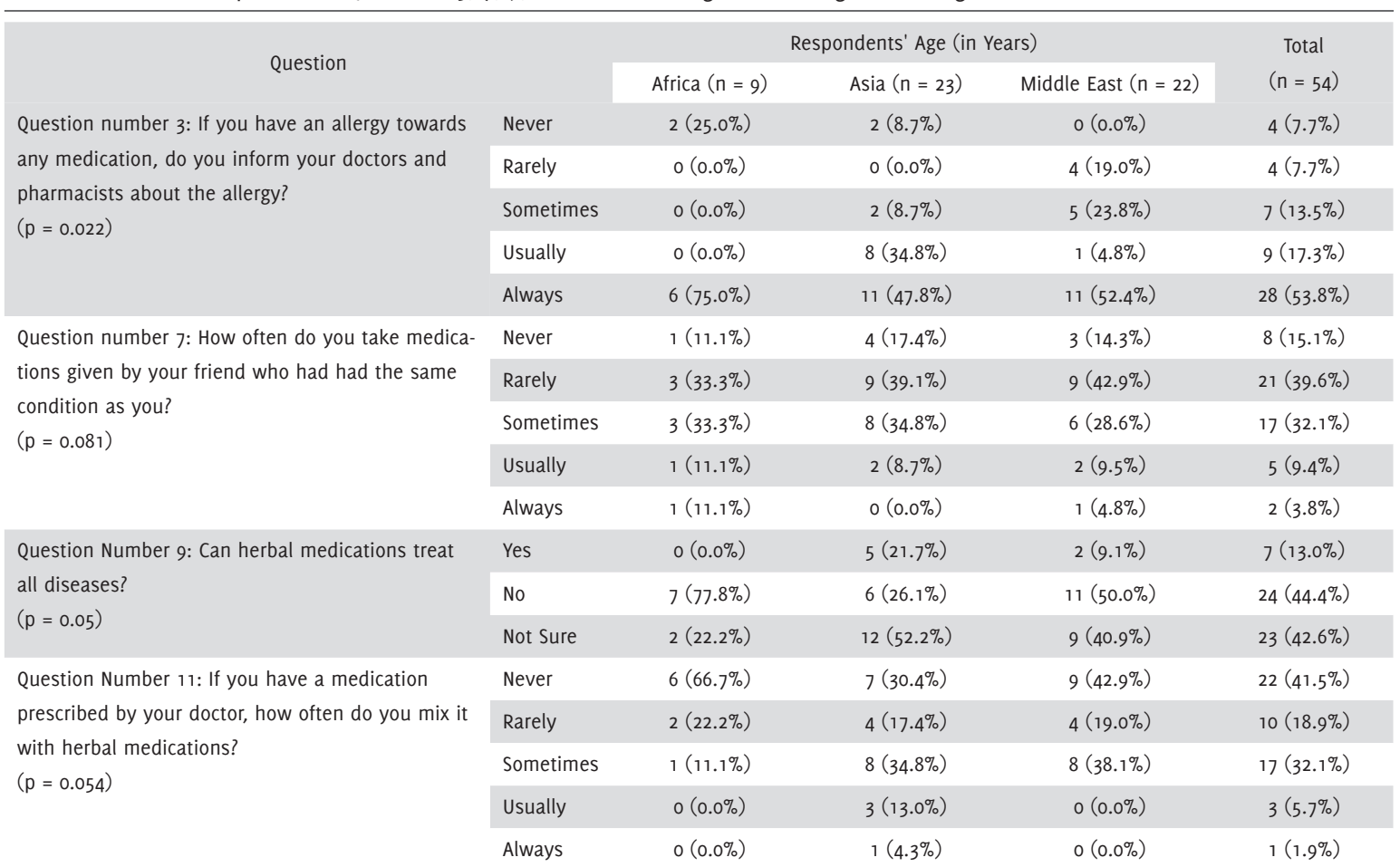

complementary and alternative medicine modalities and had used it at least once. ${ }^{8}$ In contrast, only $20-25 \%$ of Australians were able to properly use herbal medicine, as their awareness of the subject was very low. ${ }^{9}$ In Saudi Arabia, a study was conducted among Saudi pharmacy students, and it showed that they had limited knowledge regarding complementary and alternative medicine. However, this kind of therapy was widely used among these students, where one in three Saudi pharmacy students had been treated by using alternative medicine at least once in their lives, and $24 \%$ of them did not mind using it even if it is not approved clinically. Also, most of them agreed that most of the complementary and alternative medicine modalities are effective, even though $53 \%$ of them mentioned that these modalities can cause harmfulness. ${ }^{10}$ Educational background was found to have an important role in choosing herbal treatment. The findings suggested that people with lower educational backgrounds tend to favor herbal medicine over conventional medicine. Also, patients with higher education tend to have better awareness of herbal medicine and resources, such as books or the internet. It is evident that their academic background plays a major role in gaining appropriate knowledge of the subject."

For people who are unaware of proper medication use, intensive education and awareness promotion are required to decrease medication errors. ${ }^{12}$ Doctors should also take a detailed history from their patients regarding the usage of herbal medicines. According to a study conducted by Kashani et al, merely $3.2 \%$ of the research participants reported that their doctors do know about their herbal medicine intake, although most of them admitted that their physician should be aware of it." About $31 \%$ of the patients confirmed that they took herbal medicine for conditions such as infertility, digestion problems, psychological diseases and common colds. In addition, $91.8 \%$ of the consumers bought herbal medicine from traditional herbal shops and only $5.8 \%$ purchased it from a pharmacy."
In this study, there were no significant differences in answers in relation to students' educational backgrounds, in contrast to findings of a previous research which showed that academic background plays a major role in selecting herbal medicine. ${ }^{11}$ On the other hand, the region of origin of students was found to be associated with whether they alert their doctors or pharmacists to their drug allergy. This is consistent with a previous study conducted by Bamidele et al, which found that patients' knowledge and needs differ from region to region and from country to country.?

One limitation of this study is that the target of the study was international students, but there was little diversity in the nationalities of the participants. There were no students from some continents, such as Europe, North America, South America and Australia. Further research is needed to find out the attitudes and perceptions of international students from these areas towards using conventional medication and herbal medicine, so that a more thorough examination of the association between backgrounds and knowledge and attitudes towards medication use could be performed.

All in all, this study found that international students have different knowledge, attitudes and behaviors about using conventional medication and herbal medicine. Their cultures and regions were significantly associated with some of these differences. However, their age and educational backgrounds were not. It is therefore important to educate students from different regions and cultural backgrounds about the use of conventional medications and herbal medicines. Information regarding the proper use of medications and the dangers of drug interactions should be included in the curricula of formal education and disseminated to the public using culturally sensitive strategies. Further research is required to provide better insights into use of medications among students and their attitudes towards conventional and alternative medicine. 


\section{Original Article}

\section{References}

1. GU Q, Dillon CF, Burt VL. Prescription drug use continues to increase: U.S. prescription drug data for 2007-2008. NCHS data brief, no 42. Hyattsville (MD): National Center for Health Statistics; 2010.

2. Barnes PM, Bloom B, Nahin RL. Complementary and alternative medicine use among adults and children: United States, 2007. Natl Health Stat Report. 2008 Dec 10;(12):1-23.

3. Vickers KA, Jolly KB, Greenfield SM. Herbal medicine: women's views, knowledge and interaction with doctors: a qualitative study. BMC Complement Altern Med. 2006 Dec 7;6:40.

4. Onyiapat JI, Okoronkwo IL, Ogbonnaya NP. Complementary and alternative medicine use among adults in Enugu, Nigeria. BMC Complement Altern Med. 2011 Mar 4;11:19.

5. World Health Organization. The pursuit of responsible use of medicines: sharing and learning from country experiences. Geneva: World Health Organization; 2012.

6. Yekta Z, Zamani A, Mehdizade M, Farajzadegan Z. Pattern of complementary and alternative medicine use in urban population. J Res Health Sci. 2007 Jul 28;7(1):24-31.

7. Bamidele J0, Adebimpe W0, Oladele EA. Knowledge, attitude and use of al- ternative medical therapy amongst urban residents of Osun State, southwestern Nigeria. Afr J Tradit Complement Altern Med. 2009 May 7;6(3):281-8. 8. James PB, Bah AJ. Awareness, use, attitude and perceived need for Complementary and Alternative Medicine (CAM) education among undergraduate pharmacy students in Sierra Leone: a descriptive cross-sectional survey. BMC Complement Altern Med. 2014 Nov 8;14:438.

9. Stankiewicz M, Smith C, Alvino H, Norman R. The use of complementary medicine and therapies by patients attending a reproductive medicine unit in South Australia: a prospective survey. Aust N Z J Obstet Gynaecol. 2007 Apr;47(2):145-9.

10. Al-Omar HA, Al-Arifi MN. Pharmacy students' use, knowledge and attitudes toward complementary and alternative medicine at Riyadh region, Saudi Arabia. Int J Green Pharm 2011;5(1):16-23.

11. Kashani L, Hassanzadeh E, Mirzabeighi A, Akhondzadeh S. Knowledge, attitude and practice of herbal remedies in a group of infertile couples. Acta Med Iran. 2013 Apr 6;51(3):189-94.

12. Shah AP, Parmar S, Ramkishan A, Mehta AA. Knowledge, attitude and practice (KAP) survey regarding the safe use of medicine in rural area of Gujarat. Adv Trop Med Pub Health Int. 2011;1(2):66-70.

\section{Acknowledgments}

The author would like to thank Dominika and Denis for their valuable advice and comments on the writing of this manuscript and Stephen Walsh for helping out with the data analysis.

\section{Conflict of Interest Statement at Funding}

The Authors have no funding, financial relationships or conflicts of interest to disclose.

Author Contributions

Conception and design the work/idea, Collect data/obtaining results, Analysis and interpretation of data, Write the manuscript, Critical revision of the manuscript, Approval of the final version: ATE.

Cite as:

Eissa AT. Attitudes and Perceptions among Dublin International Foundation College Students towards Taking Conventional Medications and Herbal Medicines. Int J Med Students. 2015 Sep-Dec;3(3):123-6. 